\title{
Modelling and Analysis of Nonstationary Vehicle-to-Infrastructure Channels with Time-Variant Angles of Arrival
}

\author{
Matthias Pätzold $\mathbb{D}^{1}$ and Carlos A. Gutierrez $\mathbb{D D}^{2}$ \\ ${ }^{1}$ Faculty of Engineering and Science, University of Agder, 4898 Grimstad, Norway \\ ${ }^{2}$ Universidad Autonoma de San Luis Potosi, 78290 San Luis Potosi, SLP, Mexico \\ Correspondence should be addressed to Matthias Pätzold; matthias.paetzold@uia.no
}

Received 17 October 2017; Revised 16 January 2018; Accepted 4 February 2018; Published 15 March 2018

Academic Editor: Enrico M. Vitucci

Copyright (C) 2018 Matthias Pätzold and Carlos A. Gutierrez. This is an open access article distributed under the Creative Commons Attribution License, which permits unrestricted use, distribution, and reproduction in any medium, provided the original work is properly cited.

\begin{abstract}
In mobile radio channel modelling, it is generally assumed that the angles of arrival (AOAs) are independent of time. This assumption does not in general agree with real-world channels in which the AOAs vary with the position of a moving receiver. In this paper, we first present a mathematical model for the time-variant AOAs. This model serves as the basis for the development of two nonstationary multipath fading channels models for vehicle-to-infrastructure communications. The statistical properties of both channel models are analysed with emphasis on the time-dependent autocorrelation function (ACF), time-dependent mean Doppler shift, time-dependent Doppler spread, and the Wigner-Ville spectrum. It is shown that these characteristic quantities are greatly influenced by time-variant AOAs. The presented analytical framework provides a new view on the channel characteristics that goes well beyond ultra-short observation intervals over which the channel can be considered as wide-sense stationary.
\end{abstract}

\section{Introduction}

In a typical downlink scenario, where plane waves travel from a base station (BS) to a mobile station (MS) via a large number of fixed scattering objects, the angles of arrival (AOAs) of the received signals are changing along the moving route of the MS. Only for very short observation intervals in which the MS travels a few tens of the wavelengths [1], the temporal variation of the AOAs can be neglected justifying the wide-sense stationary assumption of multipath fading channels. The lengths of the stationary intervals during which the mobile radio channel can be considered as widesense stationary or quasi-stationary have been investigated (e.g., in [2-4] and the references therein). By pushing the observation interval beyond the stationary interval, the received signal captures nonstationary effects that call for new channel modelling approaches using time-frequency analysis techniques [5]. One of the effects that come with long observation intervals is that the AOAs and thus the Doppler frequencies are changing with time along the MS's moving route.
Attempts to include the temporal variations of the AOAs in mobile radio channel models have been made in [6-8]. In [6], a nonstationary multiple-input multiple-output (MIMO) vehicle-to-vehicle (V2V) channel model has been derived by assuming that the AOAs and AODs are piecewise constant. In [7], a proposal has been made for the extension of the IMTAdvanced channel model [9] by replacing the time-invariant model parameters, such as the propagation delays, AOAs, and the angels of departure (AODs) by time-variant parameters. In [8], a nonstationary one-ring model has been introduced in which the time-variant AOAs have been modelled by stochastic processes rather than random variables.

This paper is an extended version of our conference paper [10]. It expands on the recent results by studying the impact of time-variant AOAs on the statistical properties of multipath fading channels. It is shown that the multipath fading channel becomes non-wide-sense stationary if the AOAs change with time. Two new nonstationary channel models with timevariant AOAs are derived. The first one has an instantaneous channel phase that is related to the instantaneous Doppler 
frequency via the phase-frequency relationship [11], while the second one is based on a sum-of-cisoids (SOC) model in which the time-independent Doppler frequencies are replaced by time-dependent Doppler frequencies. The latter approach is simple, straightforward, and intuitive but results in a less accurate nonstationary channel model. The statistical properties of both channel models are investigated with emphasis on the time-dependent autocorrelation function (ACF), time-dependent mean Doppler shift, time-dependent Doppler spread, and the Wigner-Ville spectrum. Our analysis shows that our first proposed nonstationary channel model is consistent with respect to the mean Doppler shift and the Doppler spread, while this consistency property is not fulfilled by the SOC model with time-variant Doppler frequencies. The two proposed nonstationary channel models provide a trade-off between accuracy and complexity concerning the mathematical expressions.

One of the main differences between [6-8] and our paper is that the AOAs are modelled in different ways. For example, in [6], the AOAs are modelled as piecewise constant functions, that is, these parameters are considered as constant apart from a finite number of jumps, while in our paper the AOAs are modelled in our paper as continuous time-variant functions. Another difference is that the models in [6-8] have been developed for different propagation scenarios. The V2V channel model in [6] has been developed to simulate propagation scenarios which are typical for T-junctions. The BS-to-MS channel model in [7] covers basically the same scenarios as the IMT-Advanced channel model [9], while the model in [8] is restricted to scenarios that can be generated by the one-ring model under the assumption of isotropic scattering. This contrasts with our nonstationary generic model which is not restricted to any specific propagation scenario. The drawback of the models in [6-8] is that they are inconsistent with respect to the mean Doppler shift and the Doppler spread. Our preferred model avoids this drawback by using an integral relationship between the instantaneous channel phases and the corresponding instantaneous Doppler frequencies.

The organization of this paper is as follows. Section 2 presents the derivation of two nonstationary multipath fading channel models with time-variant AOAs. Their statistical properties will be analysed in Section 3. The numerical key results of our study are visualized in Section 4. Section 5 provides guidelines for various extensions of the model. Finally, Section 6 draws the conclusion and suggests possible future research topics in relation to the issues addressed in this paper.

\section{Derivation of the Nonstationary Multipath Channel Models}

2.1. Time-Variant AOAs. We consider a downlink non-lineof-sight (NLOS) propagation scenario in which a fixed BS operates as transmitter, and an MS acts as receiver. It is supposed that the BS and the MS are equipped with omnidirectional antennas. The BS antenna is elevated and unobstructed by any object, whereas the MS antenna is surrounded by a large number of $N$ fixed scattering objects called

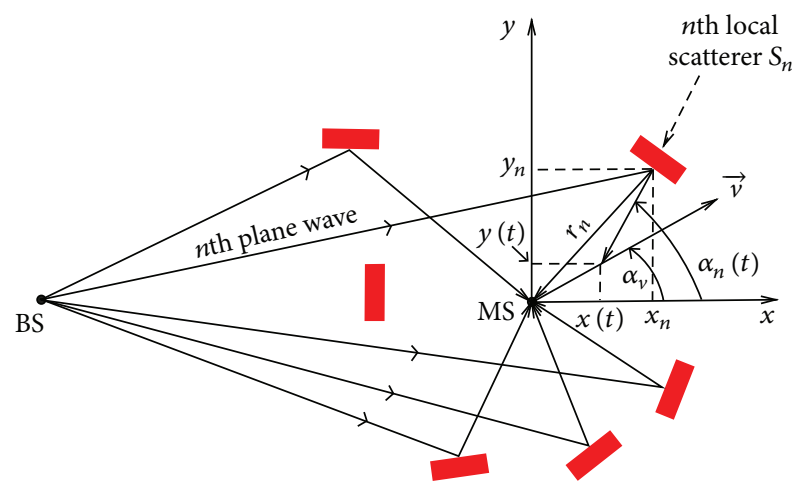

FIGURE 1: A multipath propagation scenario with time-variant AOAs $\alpha_{n}(t)$.

henceforth scatterers $S_{n}(n=1,2, \ldots, N)$. The coordinate system has been chosen such that the MS is located at the origin $(0,0)$ of the $x y$-plane at $t=0$. Furthermore, it is assumed that the MS moves with constant velocity $\vec{v}$ in the direction determined by the angle of motion $\alpha_{v}$ as indicated in Figure 1. For reasons of clarity, this figure highlights only the location of the scatterer $S_{n}$ from which the MS receives the $n$th multipath component (plane wave) $\mu_{n}(t)$ in the form of $\mu_{n}(t)=c_{n} \exp \left\{j \theta_{n}(t)\right\}$, where $c_{n}$ denotes the path gain which is supposed to be constant, and $\theta_{n}(t)$ is the associated channel phase that will be studied in Section 2.3. The corresponding AOA $\alpha_{n}(t)$ is defined as the angle between the propagation direction of the $n$th incident plane wave and the $x$-axis, that is,

$$
\alpha_{n}(t)=\operatorname{atan} 2\left(y_{n}-y(t), x_{n}-x(t)\right)
$$

for $n=1,2, \ldots, N$, where $\operatorname{atan} 2(y, x)$ denotes the fourquadrant inverse tangent function. It should be mentioned that the four-quadrant inverse tangent function atan2 $(y, x)$ returns the angle of the vector $(x, y)$ with the positive $x$-axis in the range $(-\pi, \pi)$. This function contrasts with the inverse tangent function $\operatorname{atan}(y / x)$, whose results are limited to the interval $(-\pi / 2, \pi / 2)$. In (1), the symbols $x_{n}$ and $y_{n}$ denote the coordinates of the scatterer $S_{n}$; and $x(t)$ and $y(t)$ indicate the position of the MS at time $t$. According to (1), the AOA $\alpha_{n}(t)$ is a nonlinear function of time $t$, which can be turned into a linear function by developing $\alpha_{n}(t)$ in a Taylor series around $t=0$ and retaining only the first two terms. This results in the following model for the time-variant AOA:

$$
\alpha_{n}(t)=\alpha_{n}+\gamma_{n} \cdot t
$$

where

$$
\begin{aligned}
& \alpha_{n}=\alpha_{n}(0)=\operatorname{atan} 2\left(y_{n}, x_{n}\right), \\
& \gamma_{n}=\left.\frac{d}{d t} \alpha_{n}(t)\right|_{t=0}=\frac{v}{r_{n}} \sin \left(\alpha_{n}-\alpha_{v}\right) .
\end{aligned}
$$

In (4), $r_{n}$ denotes the distance from the scatterer $S_{n}$ to the origin of the $x y$-plane, that is, $r_{n}=\sqrt{x_{n}^{2}+y_{n}^{2}}$, as can be deduced from the geometrical model in Figure 1. In Section 4, 
it is shown that the two-term Taylor series expansion of $\alpha_{n}(t)$ in (2) is sufficiently accurate for small observation intervals $T$.

2.2. Time-Variant Doppler Frequencies. Owing to the Doppler effect combined with the new feature that the AOAs $\alpha_{n}(t)$ vary with time, it follows that the $n$th incident plane wave highlighted in Figure 1 experiences a time-variant Doppler shift of $f_{n}(t)=f_{\max } \cos \left(\alpha_{n}(t)-\alpha_{v}\right)$ that can be expressed by using (2) as

$$
f_{n}(t)=f_{\max } \cos \left(\alpha_{n}-\alpha_{v}+\gamma_{n} t\right)
$$

for $n=1,2, \ldots, N$, where $f_{\max }$ stands for the maximum Doppler frequency. For a given propagation scenario with constant parameters $f_{\max }, \alpha_{n}, \alpha_{v}$, and $\gamma_{n}$, the time-variant Doppler shift $f_{n}(t)$ is a deterministic function of time. Otherwise, if one or several model parameters, for example, $\alpha_{n}$ and thus $\gamma_{n}$, are random variables, then $f_{n}(t)$ represents a stochastic process. If the MS moves during the time interval $[0, T]$, then $f_{n}(t)$ describes a curve starting from the initial Doppler frequency $f_{n}(0)=f_{\max } \cos \left(\alpha_{n}-\alpha_{v}\right)$ and ending with the finishing Doppler frequency $f_{n}(T)=f_{\max } \cos \left(\alpha_{n}-\alpha_{v}+\right.$ $\left.\gamma_{n} T\right)$.

The time-dependent mean Doppler shift $B_{f}^{(1)}(t)$ and the time-dependent Doppler spread $B_{f}^{(2)}(t)$ can be computed according to

$$
\begin{aligned}
B_{f}^{(1)}(t) & =\frac{\sum_{n=1}^{N} c_{n}^{2} f_{n}(t)}{\sum_{n=1}^{N} c_{n}^{2}}, \\
B_{f}^{(2)}(t) & =\sqrt{\frac{\sum_{n=1}^{N} c_{n}^{2} f_{n}^{2}(t)}{\sum_{n=1}^{N} c_{n}^{2}}-\left(B_{f}^{(1)}(t)\right)^{2}} .
\end{aligned}
$$

2.3. Instantaneous Channel Phase. The instantaneous channel phase $\theta_{n}(t)$ of the $n$th multipath component $\mu_{n}(t)=$ $c_{n} \exp \left\{j \theta_{n}(t)\right\}$ is related to the instantaneous Doppler frequency $f_{n}(t)$ via the phase-frequency relationship [5, Eq. $(1.3 .40)]$

$$
\theta_{n}(t)=2 \pi \int_{-\infty}^{t} f_{n}(x) d x
$$

for $n=1,2, \ldots, N$. Using (5), the instantaneous phase $\theta_{n}(t)$ can be developed as follows:

$$
\begin{aligned}
\theta_{n}(t) & =\underbrace{2 \pi \int_{-\infty}^{0} f_{n}(x) d x}_{\theta_{n}}+2 \pi \int_{0}^{t} f_{n}(x) d x \\
= & \theta_{n} \\
& +2 \pi \frac{f_{\max }}{\gamma_{n}}\left[\sin \left(\alpha_{n}-\alpha_{v}+\gamma_{n} t\right)-\sin \left(\alpha_{n}-\alpha_{v}\right)\right],
\end{aligned}
$$

where $\theta_{n}=\theta_{n}(0)$ denotes the initial phase at $t=0$. The initial phases $\theta_{n}$ are generally unknown and modelled by independent identically distributed (i.i.d.) random variables, each with uniform distribution over the interval $(0,2 \pi]$ : that is, $\theta_{n} \sim \mathcal{U}(0,2 \pi]$. Equation (9) tells us that the instantaneous phase $\theta_{n}(t)$ is not only a nonlinear function of time $t$ but also periodic with period $T_{n}=2 \pi / \gamma_{n}$ if the AOA $\alpha_{n}(t)$ varies with time according to (2). In the limit $\gamma_{n} \rightarrow 0$, however, it can be shown by applying L'Hôpital's rule to (9) that

$$
\lim _{\gamma_{n} \rightarrow 0} \theta_{n}(t)=\theta_{n}+2 \pi f_{n} t
$$

where $f_{n}=f_{n}(0)=f_{\max } \cos \left(\alpha_{n}-\alpha_{v}\right)$. This result reveals a linear relationship between the instantaneous phase $\theta_{n}(t)$ and time $t$, which holds only for constant AOAs $\alpha_{n}(t)=$ $\alpha_{n}$. It should be noticed that the expression in (10) can be identified as the standard phase term of SOC channel models for Rayleigh/Rice fading channels [12, Section 4.5].

A simpler but less accurate expression than (9) can be obtained for the instantaneous phase $\theta_{n}(t)$ by developing $\theta_{n}(t)$ in a first-order Taylor series around $t=0$ as follows:

$$
\begin{aligned}
\theta_{n}(t) & \approx \theta_{n}(0)+\theta_{n}^{\prime}(0) t=\theta_{n}+2 \pi f_{\max } \cos \left(\alpha_{n}-\alpha_{v}\right) t \\
& =\theta_{n}+2 \pi f_{n} t,
\end{aligned}
$$

where $\theta_{n}^{\prime}(0)$ denotes the time derivative of $\theta_{n}(t)$ at $t=0$. By comparing the last two equations, we can conclude that the linear phase term $\theta_{n}(t)=\theta_{n}+2 \pi f_{n} t$ can be obtained from the nonlinear phase term $\theta_{n}(t)$ [see (9)] either in the limit $\gamma_{n} \rightarrow$ 0 or by developing the nonlinear phase $\theta_{n}(t)$ in a first-order Taylor series around $t=0$.

2.4. Complex Channel Gain. A model for the complex channel gain, denoted by $\mu(t)$, of a narrowband multipath fading channel is obtained by the superposition of all $N$ plane wave components $\mu_{n}(t)=c_{n} \exp \left\{j \theta_{n}(t)\right\}$, that is,

$$
\mu(t)=\sum_{n=1}^{N} c_{n} e^{j \theta_{n}(t)} .
$$

Substituting the instantaneous channel phase $\theta_{n}(t)$ according to (9) in (12) results in the complex channel gain of the proposed nonstationary multipath fading channel with timevariant AOAs

$$
\mu(t)=\sum_{n=1}^{N} c_{n} e^{j\left\{2 \pi\left(f_{\max } / \gamma_{n}\right)\left[\sin \left(\alpha_{n}-\alpha_{v}+\gamma_{n} t\right)-\sin \left(\alpha_{n}-\alpha_{v}\right)\right]+\theta_{n}\right\}} .
$$

On the other hand, starting from the SOC model for Rayleigh fading channels [12, Eq. (4.97)] and replacing there intuitively the time-independent Doppler frequencies $f_{n}$ by the instantaneous Doppler frequencies $f_{n}(t)$ according to (5) provide the complex channel gain $\mu(t)$ in a much simpler form, namely,

$$
\mu(t)=\sum_{n=1}^{N} c_{n} e^{j\left(2 \pi f_{n}(t) \cdot t+\theta_{n}\right)}
$$

This intuitive mathematical manipulation results in a nonstationary channel model that is inconsistent with respect to the mean Doppler shift $B_{f}^{(1)}(t)$ and the Doppler spread 
$B_{f}^{(2)}(t)$, as we will see in Section 3.2. Although the expression in (14) is mathematically simpler than the one in (13), the difference is not significant in terms of implementation costs and simulation time.

From the discussions in the previous subsection, it can be summed up that the two complex channel gains $\mu(t)$ in (13) and (14) include the original SOC model [13]

$$
\mu(t)=\sum_{n=1}^{N} c_{n} e^{j\left(2 \pi f_{n} t+\theta_{n}\right)}
$$

as a special case that arises if the AOA $\alpha_{n}(t)$ is supposed to be either constant $\left(\gamma_{n}=0\right)$ or if the instantaneous phase $\theta_{n}(t)$ in (12) is approximated by a first-order Taylor series [see (11)]. The main difference between the three stochastic channel models above is that the former two are non-wide-sense stationary, whereas the third one is wide-sense stationary. The statistical properties of the SOC model have been studied in [13], while those of the new non-wide-sense stationary models will be analysed in the next section.

\section{Analysis of the Nonstationary Multipath Channel Models}

3.1. Time-Dependent ACF. The time-dependent ACF $\mathscr{R}_{\mu}(\tau$, $t$ ) of a complex stochastic process $\mu(t)$ is defined as

$$
\mathscr{R}_{\mu}(\tau, t)=E\left\{\mu\left(t+\frac{\tau}{2}\right) \mu^{*}\left(t-\frac{\tau}{2}\right)\right\},
$$

where $E\{\cdot\}$ denotes the expectation operator and $(\cdot)^{*}$ stands for the complex conjugation operator. In the Appendix, it is proved that the time-dependent ACF $\mathscr{R}_{\mu}(\tau, t)$ of the complex channel gain $\mu(t)$ in (13) can be written as

$$
\mathscr{R}_{\mu}(\tau, t)=\sum_{n=1}^{N} c_{n}^{2} e^{j 2 \pi f_{n}(t) \cdot \operatorname{sinc}\left(\gamma_{n} \tau / 2\right) \cdot \tau},
$$

where $\operatorname{sinc}(\cdot)$ denotes the sinc function, which is defined by $\operatorname{sinc}(x)=\sin (x) / x$.

Analogously, it can be shown that the time-dependent ACF $\mathscr{R}_{\mu}(\tau, t)$ of the complex channel gain $\mu(t)$ introduced in (14) can be expressed by

$$
\mathscr{R}_{\mu}(\tau, t)=\sum_{n=1}^{N} c_{n}^{2} e^{j 2 \pi\left[f_{n}(t) \cos \left(\gamma_{n}(\tau / 2)\right)+f_{n}^{\prime}(t) \operatorname{sinc}\left(\gamma_{n}(\tau / 2)\right) t\right] \tau},
$$

where $f_{n}(t)$ is the time-variant Doppler shift in (5) and $f_{n}^{\prime}(t)$ denotes its derivative with respect to time $t$.

For the special case that the AOA $\alpha_{n}(t)$ is constant, that is, $\gamma_{n}=0$, it is obvious that the two time-dependent ACFs in (17) and (18) reduce to

$$
\mathscr{R}_{\mu}(\tau)=\mathscr{R}_{\mu}(\tau, t)=\sum_{n=1}^{N} c_{n}^{2} e^{j 2 \pi f_{n} \tau}
$$

which represents the ACF of the SOC model described by (15). In this case, the ACF depends only on the time separation $\tau$ but not on time $t$, which was to be expected, because the SOC process $\mu(t)$ is wide-sense stationary.

Furthermore, if $\gamma_{n}=0$ and $\alpha_{n} \sim \mathcal{U}(0,2 \pi]$, then the expressions in (17)-(19) reduce to the ACF $\mathscr{R}_{\mu}(\tau)=$ $2 \sigma_{0}^{2} J_{0}\left(2 \pi f_{\max } \tau\right)$, where $2 \sigma_{0}^{2}=\sum_{n=1}^{N} c_{n}^{2}$ denotes the mean power of the complex channel gain $\mu(t)$, and $J_{0}(\cdot)$ is the zeroth-order Bessel function of the first kind [14, Eq. (8.4111)]. In other words, the proposed nonstationary multipath fading channel models include the classical Jakes/Clarke model $[1,15]$ as a special case.

3.2. Time-Dependent Mean Doppler Shift and Time-Dependent Doppler Spread. From the time-dependent ACF $\mathscr{R}_{\mu}(\tau, t)$, the time-dependent mean Doppler shift $B_{\mu}^{(1)}(t)$ and the timedependent Doppler spread $B_{\mu}^{(2)}(t)$ can be derived by means of

$$
\begin{aligned}
& B_{\mu}^{(1)}(t)=\frac{1}{2 \pi j} \frac{\dot{\mathscr{R}}_{\mu}(0, t)}{\mathscr{R}_{\mu}(0, t)}, \\
& B_{\mu}^{(2)}(t)=\frac{1}{2 \pi} \sqrt{\left(\frac{\mathscr{\mathscr { R }}_{\mu}(0, t)}{\mathscr{R}_{\mu}(0, t)}\right)^{2}-\frac{\ddot{\mathscr{R}}_{\mu}(0, t)}{\mathscr{R}_{\mu}(0, t)}},
\end{aligned}
$$

respectively, where $\dot{\mathscr{R}}_{\mu}(0, t)\left(\ddot{\mathscr{R}}_{\mu}(0, t)\right)$ denotes the first (second) order derivative of $\mathscr{R}_{\mu}(\tau, t)$ with respect to $\tau$ at $\tau=0$. Inserting (17) in (20) and (21) results after some straightforward mathematical steps in the following closed-form solutions:

$$
\begin{aligned}
& B_{\mu}^{(1)}(t)=\frac{\sum_{n=1}^{N} c_{n}^{2} f_{n}(t)}{\sum_{n=1}^{N} c_{n}^{2}}, \\
& B_{\mu}^{(2)}(t)=\sqrt{\frac{\sum_{n=1}^{N} c_{n}^{2} f_{n}^{2}(t)}{\sum_{n=1}^{N} c_{n}^{2}}-\left(B_{\mu}^{(1)}(t)\right)^{2}} .
\end{aligned}
$$

A comparison of (22) with (6) and (23) with (7) reveals that the equalities $B_{\mu}^{(1)}(t)=B_{f}^{(1)}(t)$ and $B_{\mu}^{(2)}(t)=B_{f}^{(2)}(t)$ hold, from which we can conclude that the proposed nonstationary multipath fading channel model described by (13) is consistent with respect to both the mean Doppler shift and the Doppler spread.

On the other hand, if we insert (18) in (20) and (21), then we obtain

$$
\begin{aligned}
& B_{\mu}^{(1)}(t)=\frac{\sum_{n=1}^{N} c_{n}^{2}\left(f_{n}(t)+f_{n}^{\prime}(t) \cdot t\right)}{\sum_{n=1}^{N} c_{n}^{2}}, \\
& B_{\mu}^{(2)}(t)=\sqrt{\frac{\sum_{n=1}^{N} c_{n}^{2}\left(f_{n}(t)+f_{n}^{\prime}(t) \cdot t\right)^{2}}{\sum_{n=1}^{N} c_{n}^{2}}-\left(B_{\mu}^{(1)}(t)\right)^{2} .}
\end{aligned}
$$

This result demonstrates that the simple nonstationary channel model introduced in (14) is inconsistent with respect to the mean Doppler shift and the Doppler spread, because $B_{\mu}^{(1)}(t) \neq B_{f}^{(1)}(t)$ and $B_{\mu}^{(2)}(t) \neq B_{f}^{(2)}(t)$ hold. Concerning 
the SOC process $\mu(t)$ in (15), we mention for completeness that the equalities $B_{\mu}^{(1)}=B_{f}^{(1)}$ and $B_{\mu}^{(2)}=B_{f}^{(2)}$ hold, where $B_{\mu}^{(1)}$ and $B_{\mu}^{(2)}$ are the same quantities as in (22) and (23), respectively, if we replace $f_{n}(t)$ by $f_{n}$. Thus, the SOC model is consistent with respect to the mean Doppler shift and the Doppler spread. More information on the consistency of nonstationary multipath fading channels can be found in [16].

3.3. Wigner-Ville Spectrum. The Wigner-Ville spectrum, which is also called the time-varying spectrum or the evolutive spectrum, will be denoted by $\mathcal{S}_{\mu}(f, t)$. This function is defined as the Fourier transform of the time-dependent ACF $\mathscr{R}_{\mu}(\tau, t)$ with respect to $\tau$ [11]: that is,

$$
\mathcal{S}_{\mu}(f, t)=\int_{-\infty}^{\infty} \mathscr{R}_{\mu}(\tau, t) e^{-j 2 \pi f t} d \tau
$$

Inserting (17) in (26) and using the property $\mathscr{R}_{\mu}(\tau, t)=$ $\mathscr{R}_{\mu}^{*}(-\tau, t)$, we can express the Wigner-Ville spectrum $\mathcal{S}_{\mu}(f, t)$ of the proposed nonstationary multipath fading channel model described by (13) as

$$
\begin{aligned}
& \mathcal{S}_{\mu}(f, t) \\
& \quad=2 \sum_{n=1}^{N} c_{n}^{2} \int_{0}^{\infty} \cos \left\{2 \pi\left[f-f_{n}(t) \operatorname{sinc}\left(\gamma_{n} \frac{\tau}{2}\right)\right] \tau\right\} d \tau .
\end{aligned}
$$

For the wide-sense stationary case, for which $\gamma_{n}=0$ holds, the Wigner-Ville spectrum $\mathcal{S}_{\mu}(f, t)$ in (27) reduces to the Doppler power spectral density (PSD) of the SOC process $\mu(t)$ presented in (15), that is,

$$
\delta_{\mu}(f)=\sum_{n=1}^{N} c_{n}^{2} \delta\left(f-f_{n}\right)
$$

Furthermore, for the isotropic scattering case, in which $c_{n}$ and $\alpha_{n}$ are i.i.d. random variables with $E\left\{c_{n}^{2}\right\}=2 \sigma_{0}^{2} / N$ and $\alpha_{n} \sim \mathcal{U}(0,2 \pi]$, we obtain the Jakes/Clarke PSD [1,15] after computing the expected value of $\mathcal{S}_{\mu}(f)$ in (28). Hence, the Wigner-Ville spectrum $\mathcal{S}_{\mu}(f, t)$ in $(27)$ includes the classical Jakes/Clarke Doppler spectrum as a special case.

\section{Numerical Results}

This section presents a selection of numerical results to illustrate the main findings of this paper. In all considered propagation scenarios, we have set the number of multipath components $N$ to $N=10$. The gains $c_{n}$ and initial AOAs $\alpha_{n}=\alpha_{n}(0)$ have been computed by using the extended method of exact Doppler spread (EMEDS) [17]. According to this method, the parameters $c_{n}$ and $\alpha_{n}$ are given by

$$
\begin{aligned}
c_{n} & =\sigma_{0} \sqrt{\frac{2}{N}}, \\
\alpha_{n} & =\frac{2 \pi}{N}\left(n-\frac{1}{4}\right),
\end{aligned}
$$

respectively, and the initial phases $\theta_{n}=\theta_{n}(0)$ are considered as realizations of independent random variables, each characterized by a uniform distribution over the interval $(0,2 \pi]$. If

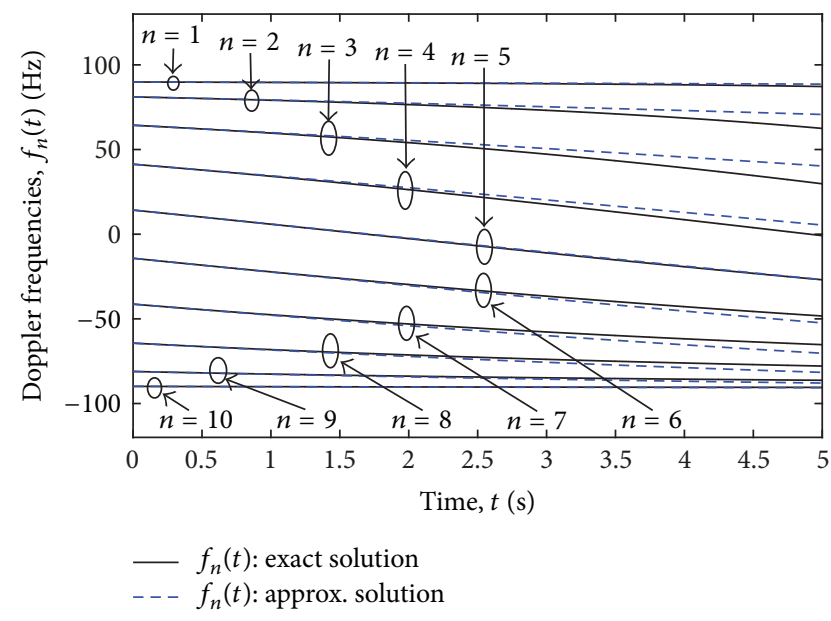

Figure 2: Trend of the time-variant Doppler frequencies $f_{n}(t)(n=$ $1,2, \ldots, N)$ by using the exact solution (black solid line) and the approximate solution (blue dashed line), where $N=10$.

not stated otherwise, the radii $r_{n}$ in Figure 1 have been set to $50 \mathrm{~m}$ for all $n=1,2, \ldots, N$. For the mean power (variance) $\sigma_{0}^{2}$ of the in-phase and quadrature components of $\mu(t)$, we have chosen the value $\sigma_{0}^{2}=1$. The carrier frequency was set to $5.9 \mathrm{GHz}$, and the maximum Doppler frequency $f_{\max }$ was supposed to be $f_{\max }=91 \mathrm{~Hz}$. This corresponds to a mobile speed of $v=|\vec{v}|=16.65 \mathrm{~km} / \mathrm{h}$, where we have assumed that the MS moves in $x$-direction, implying that the angle of motion $\alpha_{v}$ equals zero, that is, $\alpha_{v}=0$.

Figure 2 depicts the trend of the time-variant Doppler frequencies $f_{n}(t)$ by using the exact expression for the AOAs $\alpha_{n}(t)$ according to (1). For comparison, this figure also shows the behaviour of $f_{n}(t)$ for the approximate solution of $\alpha_{n}(t)$ in (2). Figure 2 shows clearly that the first-order approximation is quite good over the interval from 0 to $2.162 \mathrm{~s}$ during which the MS has covered a distance of $10 \mathrm{~m}$.

Figure 3 illustrates the signal envelope $|\mu(t)|$ by using the SOC model [see (15), Case I], the proposed nonstationary multipath fading channel model [see (13), Case II], and the simple nonstationary model [see (14), Case III]. This figure demonstrates clearly that the temporal variations of the AOAs $\alpha_{n}(t)$ have a great influence on the temporal behaviour of the signal envelope $|\mu(t)|$. It is interesting to note that the three signal envelopes are identical at $t=0$ and very similar for small values of $t>0$, but they differ considerably with increasing values of $t$. It should be mentioned that different realizations of the initial phases $\theta_{n}=\theta_{n}(0)$ result in different sample functions of the signal envelopes, but the aforementioned trend is the same for all realizations.

Figures 4 and 5 present the ACF $\mathscr{R}_{\mu}(\tau)$ of the SOC process $\mu(t)$ in (15) and the time-dependent ACF $\mathscr{R}_{\mu}(\tau, t)$ of the nonstationary process $\mu(t)$ in (13), respectively. It can be observed that both ACFs are identical at the origin $t=0$, but the temporal correlation properties of the nonstationary model differ more and more if time $t$ proceeds. This means that the temporal variations of $\alpha_{n}(t)$ influence greatly the fading behaviour of the signal envelope $|\mu(t)|$. 


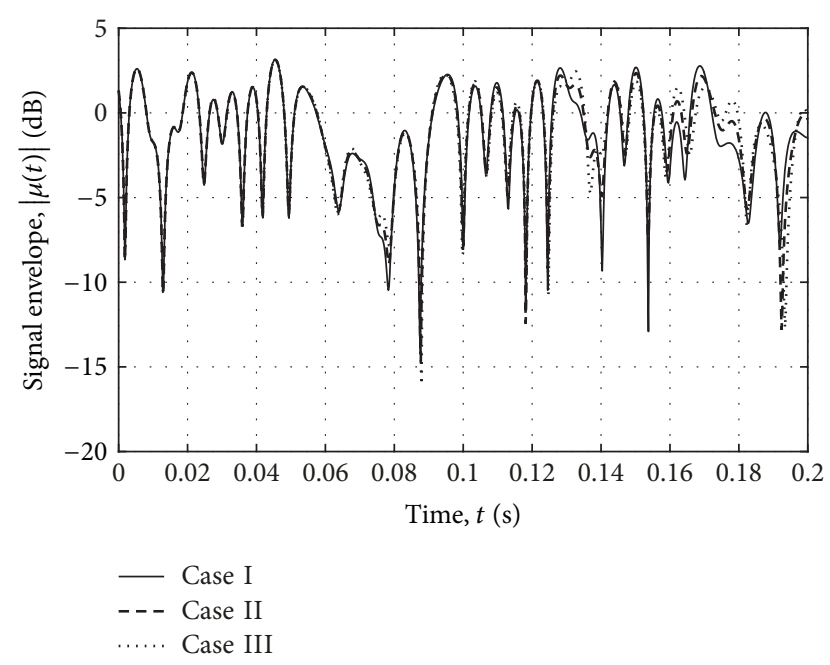

Figure 3: Illustration of the signal envelope $|\mu(t)|$ of a sample function of a wide-sense stationary SOC process [see (15)] in comparison with the signal envelopes $|\mu(t)|$ of the nonstationary processes described by (13) and (14).

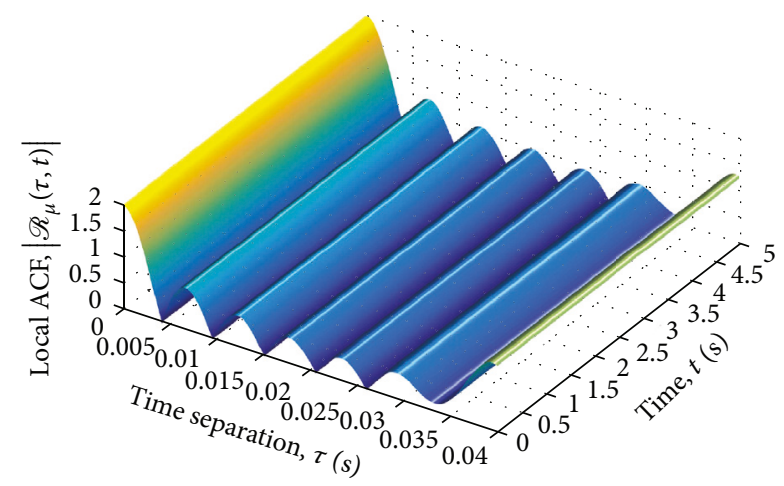

FIgURE 4: ACF $\mathscr{R}_{\mu}(\tau)=\mathscr{R}_{\mu}(\tau, t)$ of a SOC process $\mu(t)$ with constant AOAs $\alpha_{n}$ for $N=10$.

Figures 6 and 7 depict the corresponding Doppler PSD $\mathcal{S}_{\mu}(f)$ [see (28)] of the SOC process $\mu(t)$ in (15) and the Wigner-Ville spectrum $\delta_{\mu}(f, t)$ [see (27)] of the nonstationary process $\mu(t)$ in (13), respectively. A comparison of the two spectral representations shows clearly that the influence of the time-variant AOAs $\alpha_{n}(t)$ cannot be neglected. This statement is obvious as the Doppler frequencies of the Wigner-Ville spectrum (Doppler PSD) associated with the stationary SOC process remain constant over time $t$ (see Figure 6), while the spectral components of the nonstationary process experience a drift if time $t$ proceeds (see Figure 7). Finally, we mention that the results in Figure 7 have been obtained numerically by setting the upper limit of $\tau$ in the integral of (27) to $\tau=\tau_{\max }=$ $1 \mathrm{~s}$ and evaluating the integral by considering $N_{s}=1000$ samples, which results in a resolution of $\tau_{\max } / N_{s}=0.001 \mathrm{~s}$.

Figures 8 and 9 are devoted to a study on the influence of the (ring) radii $r_{n}$ on the time-dependent mean Doppler shift $B_{\mu}^{(1)}(t)$ and the time-dependent Doppler spread $B_{\mu}^{(2)}(t)$, respectively. The presented graphs show that the smaller the

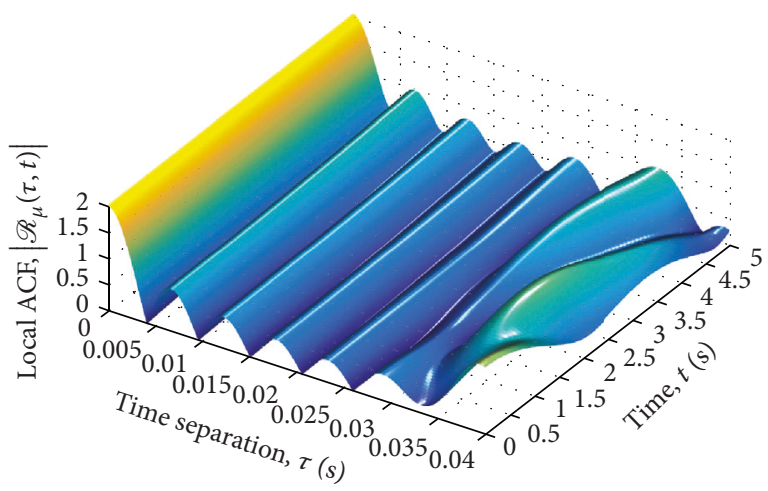

Figure 5: Time-dependent ACF $\mathscr{R}_{\mu}(\tau, t)$ of the proposed nonstationary process $\mu(t)$ with time-variant AOAs $\alpha_{n}(t)$ for $N=10$.

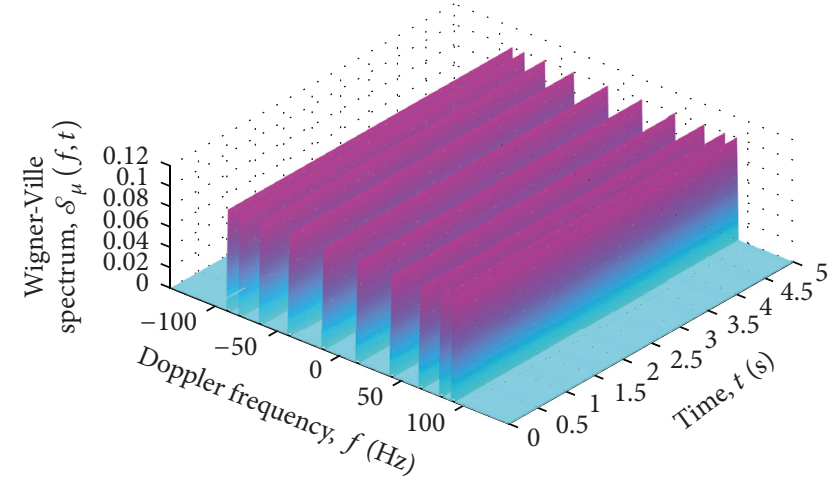

FIGURE 6: Wigner-Ville spectrum (Doppler PSD) $\mathcal{S}_{\mu}(f)=\mathcal{S}_{\mu}(f, t)$ of an SOC process $\mu(t)$ with constant AOAs $\alpha_{n}$ for $N=10$.

radii $r_{n}$ are, the faster the functions $B_{\mu}^{(1)}(t)$ and $B_{\mu}^{(2)}(t)$ are changing over time $t$. Figure 8 shows also a comparison between the time-dependent mean Doppler shift $B_{\mu}^{(1)}(t)$ of the consistent model described by (14) and the inconsistent model according to (15). Both models have the same mean Doppler shift $B_{\mu}^{(1)}(t)$ at the origin $t=0$, but the mean Doppler shifts deviate considerably from each other with increasing values of time $t$. The same statement holds for the timedependent Doppler spread shown in Figure 9. These results underline the importance of consistency, as the deviations between (22) and (24) as well as between (23) and (25) cannot be neglected.

\section{Model Extensions}

To isolate the effect of time-variant AOAs on the Doppler characteristic, we have assumed that the channel is frequency-nonselective and that the transmitter and receiver are equipped with single omnidirectional antennas. To relax these assumptions, we will provide some guidelines on model extensions in the following subsections.

5.1. Extension to Frequency-Selectivity. Starting from the narrowband multipath fading channel model in (12) and taking into account the fact that the $n$th plane wave component 


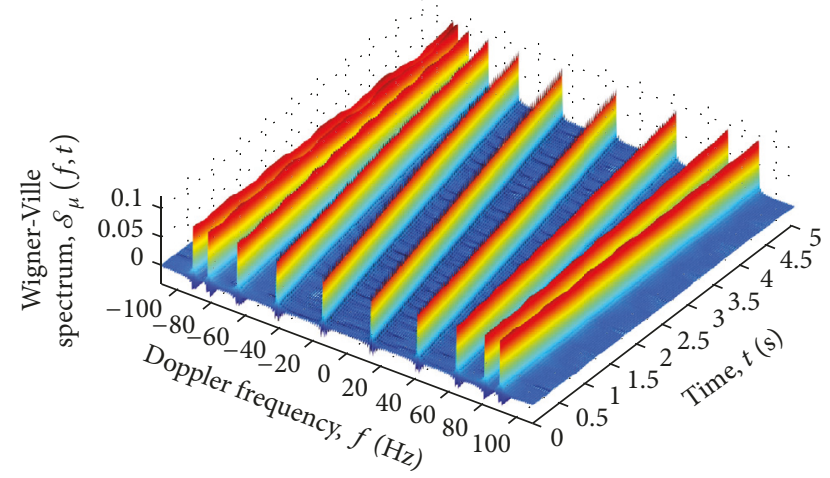

FIgURE 7: Wigner-Ville spectrum $\mathcal{S}_{\mu}(f, t)$ of the proposed nonstationary process $\mu(t)$ with time-variant AOAs $\alpha_{n}(t)$ for $N=10$.

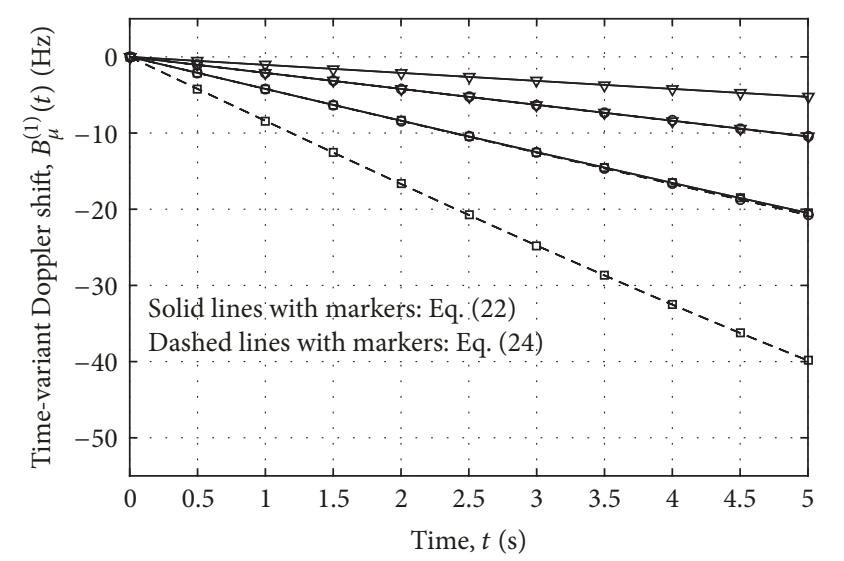

$\begin{array}{ll}\text { } & r_{n}=50 \mathrm{~m} \\ \text { ○ } & r_{n}=100 \mathrm{~m} \\ \nabla & r_{n}=200 \mathrm{~m}\end{array}$

Figure 8: Time-dependent mean Doppler shift $B_{\mu}^{(1)}(t)$ of the nonstationary channel models for different values of the radii $r_{n}$.

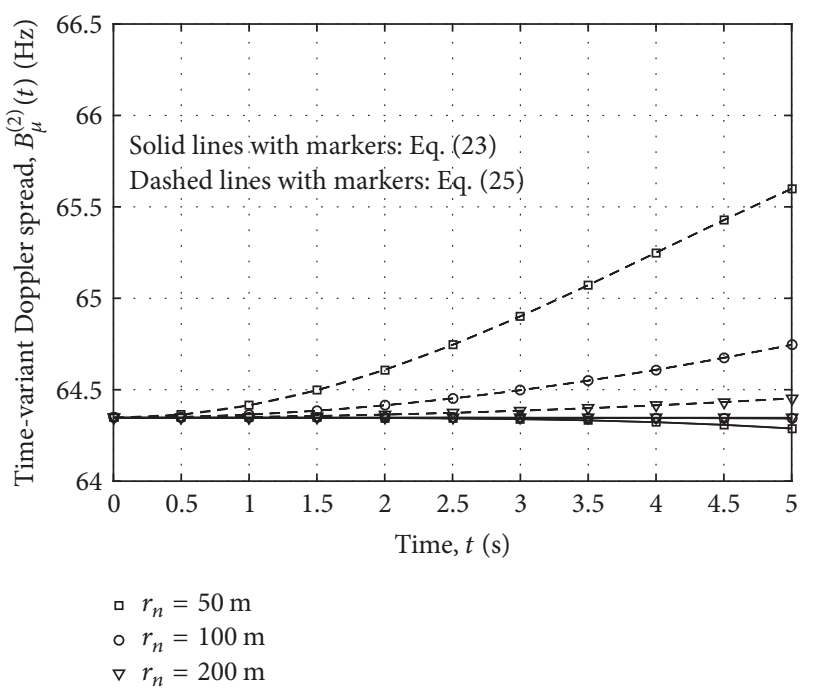

Figure 9: Time-dependent Doppler spread $B_{\mu}^{(2)}(t)$ of the nonstationary channel models for different values of the radii $r_{n}$ for $n=$ $1,2, \ldots, N(N=10)$. $\mu_{n}(t)=c_{n} \exp \left\{j \theta_{n}(t)\right\}$ will be received after a time-variant propagation delay denoted by $\tau_{n}^{\prime}(t)$, the impulse response $h\left(\tau^{\prime}, t\right)$ of the resulting non-wide-sense stationary singleinput single-output channel model can be expressed as

$$
h\left(\tau^{\prime}, t\right)=\sum_{n=1}^{N} c_{n} e^{j \theta_{n}(t)} \delta\left(\tau^{\prime}-\tau_{n}^{\prime}(t)\right)
$$

where $\theta_{n}(t)$ is given by (9) for the consistent model or by $\theta_{n}(t)=2 \pi f_{n}(t) t+\theta_{n}$ for the inconsistent model. The timevariant delay $\tau_{n}^{\prime}(t)$ can be derived from the geometrical model in Figure 1 as

$$
\tau_{n}^{\prime}(t)=\frac{1}{c_{0}}\left[\sqrt{\left(D+x_{n}\right)^{2}+y_{n}^{2}}+r_{n}(t)\right],
$$

where $c_{0}$ denotes the speed of light, $D$ is the distance between the BS and MS, and $r_{n}(t)=\sqrt{\left(x_{n}-x(t)\right)^{2}+\left(y_{n}-y(t)\right)^{2}}$.

5.2. Extension to MIMO. Let $h_{k \ell}\left(\tau^{\prime}, t\right)$ denote the impulse response of a frequency-selective MIMO channel with $M_{T}$ transit and $M_{R}$ receive antennas; then the propagation link from the $\ell$ th transmit antenna to the $k$ th receive antenna can be modelled as

$$
h_{k \ell}\left(\tau^{\prime}, t\right)=\sum_{n=1}^{N} g_{k \ell_{n}}(t) e^{j \theta_{n}(t)} \delta\left(\tau^{\prime}-\tau_{n}^{\prime}(t)\right)
$$

for $k=1,2, \ldots, M_{R}$ and $\ell=1,2, \ldots, M_{T}$, where $g_{k \ell n}(t)$ is the same as in [18, Eq. (9)] apart from the fact that we have to replace there the time-invariant quantities $r_{n}$ and $\alpha_{n}^{R}$ by timevariant quantities $r_{n}(t)$ and $\alpha_{n}^{R}(t)$, respectively. The expression in (33) can be derived by applying the design steps of the generalized principle of deterministic channel modelling [12, Section 8.1].

5.3. Other Model Extensions. The proposed model is only applicable to omnidirectional antennas. The extension to directional antennas is possible through a proper adjustment of the constant path gains $c_{n}$, which have to be replaced by time-variant path gains $c_{n}(t)$. The temporal characteristics of $c_{n}(t)$ depend on the antenna pattern and the direction in which the MS moves. It is also possible to consider birthand-death effects of the scatterers. This extension results in a multiplication of each multipath component by a birthand-death process or, equivalently, by replacing the timeinvariant path gains $c_{n}$ by proper time-variant path gains $c_{n}(t)$. The analysis of the Wigner-Ville spectrum of nonstationary mobile radio channels with time-variant path gains $c_{n}(t)$ is substantially different from the analysis in Section 3.3 and beyond the scope of this paper.

\section{Conclusion}

In this paper, we have developed and analysed multipath fading channel models with time-variant AOAs. Our study has shown that the effect of time-variant AOAs results 
in a non-wide-sense stationary multipath fading channel model. Expressions have been derived for the timedependent ACF, time-dependent mean Doppler shift, timedependent Doppler spread, and the Wigner-Ville spectrum of the proposed non-wide-sense stationary channel model. By comparing these statistical quantities with known results of studies assuming constant AOAs, we can conclude that the assumption of constant AOAs is only justified for very short observation intervals. The proposed nonstationary channel model allows extending the observation interval over a wider range without losing accuracy. The price for this added accuracy is a higher degree of complexity concerning the mathematical expressions.

One of the remaining problems that might be tackled in an upcoming study is to develop quantitative methods for the investigation of the length of the observation interval over which the proposed nonstationary channel models are sufficiently accurate. Another topic could be to extend the presented framework to the modelling of MIMO channels with time-dependent AOAs.

\section{Appendix}

\section{Derivation of the Time-Dependent ACF $\mathscr{R}_{\mu}(\tau, t)$ in (17)}

Substituting (13) in the definition of the time-dependent ACF $\mathscr{R}_{\mu}(\tau, t)=E\left\{\mu(t+\tau / 2) \mu^{*}(t-\tau / 2)\right\}$ gives

$$
\begin{aligned}
& \mathscr{R}_{\mu}(\tau, t) \\
& =E\left\{\sum_{n=1}^{N} \sum_{n=1}^{N} c_{n} c_{m} e^{j\left\{2 \pi\left(f_{\max } / \gamma_{n}\right)\left[\sin \left(\alpha_{n}-\alpha_{v}+\gamma_{n}(t+\tau / 2)\right)-\sin \left(\alpha_{n}-\alpha_{v}\right)\right]+\theta_{n}\right\}}\right. \\
& \left.\cdot e^{-j\left\{2 \pi\left(f_{\max } / \gamma_{m}\right)\left[\sin \left(\alpha_{m}-\alpha_{v}+\gamma_{m}(t-\tau / 2)\right)-\sin \left(\alpha_{m}-\alpha_{v}\right)\right]+\theta_{m}\right\}}\right\}
\end{aligned}
$$

Using $E\left\{e^{j\left(\theta_{n}-\theta_{m}\right)}\right\}=1$ if $n=m$ and 0 if $n \neq m$, we obtain

$$
\begin{gathered}
\mathscr{R}_{\mu}(\tau, t)=\sum_{n=1}^{N} c_{n}^{2} e^{j 2 \pi\left(f_{\max } / \gamma_{n}\right) \sin \left(\alpha_{n}-\alpha_{v}+\gamma_{n}(t+\tau / 2)\right)} \\
\cdot e^{-j 2 \pi\left(f_{\max } / \gamma_{n}\right) \sin \left(\alpha_{n}-\alpha_{v}+\gamma_{n}(t-\tau / 2)\right)} \\
=\sum_{n=1}^{N} c_{n}^{2} e^{j 2 \pi\left(f_{\max } /\left(\gamma_{n} / 2\right)\right) \cos \left(\alpha_{n}-\alpha_{v}+\gamma_{n} t\right) \sin \left(\gamma_{n}(\tau / 2)\right)} \\
=\sum_{n=1}^{N} c_{n}^{2} e^{j 2 \pi f_{n}(t) \operatorname{sinc}\left(\gamma_{n} \tau / 2\right) \tau}
\end{gathered}
$$

where we have used the sinc function defined as $\operatorname{sinc}(x)=$ $\sin (x) / x$.

\section{Conflicts of Interest}

The authors declare that there are no conflicts of interest regarding the publication of this paper.

\section{References}

[1] W. C. Jakes, Ed., Microwave Mobile Communications, IEEE Press, Piscataway, NJ, USA, 1994.

[2] R. He, O. Renaudin, V.-M. Kolmonen et al., "Characterization of quasi-stationarity regions for vehicle-to-vehicle radio channels," IEEE Transactions on Antennas and Propagation, vol. 63, no. 5, pp. 2237-2251, 2015.

[3] D. Umansky and M. Pätzold, "Stationarity test for wireless communication channels," in Proceedings of the 2009 IEEE Global Telecommunications Conference, GLOBECOM 2009, Honolulu, Hawaii, USA, December 2009.

[4] A. Ispas, C. Schneider, G. Ascheid, and R. Thomä, "Analysis of the local quasi-stationarity of measured dual-polarized MIMO channels," IEEE Transactions on Vehicular Technology, vol. 64, no. 8, pp. 3481-3493, 2015.

[5] B. Boashash, Ed., Time-Frequency Signal, Analysis and Processing: A Comprehensive Reference, Elsevier Academic Press, 2nd edition edition, 2015.

[6] A. Chelli and M. Pätzold, "A non-stationary MIMO vehicleto-vehicle channel model based on the geometrical T-junction model," in Proceedings of the 2009 International Conference on Wireless Communications and Signal Processing, WCSP 2009, Nanjing, China, November 2009.

[7] Z. Zhu, Y. Zhu, T. Zhang, and Z. Zeng, "A time-variant MIMO channel model based on the IMT-Advanced channel model," in Proceedings of the 2012 International Conference on Wireless Communications and Signal Processing, WCSP 2012, Huangshan, China, 2012.

[8] A. Borhani and M. Pätzold, "A non-stationary one-ring scattering model," in Proceedings of the 2013 IEEE Wireless Communications and Networking Conference, WCNC 2013, pp. 2661-2666, Shanghai, China, April 2013.

[9] ITU-R Rep. M2135, "Guidelines for evaluation of radio interface technologies for IMT-Advanced," 2008, https://www.itu.int/ dms_pub/itu-r/opb/rep/R-REP-M.2135-2008-PDF-E.pdf.

[10] M. Pätzold and C. A. Gutiérrez, "Modelling and analysis of nonstationary multipath fading channels with time-variant angles of arrival," in Proceedings of the 2017 IEEE 85th Vehicular Technology Conference (VTC Spring), Sydney, Australia, June 2017.

[11] W. Martin, "Time-frequency analysis of random signals," in Proceedings of the IEEE International Conference on Acoustics, Speech, and Signal Processing, vol. 3, pp. 1325-1328, Paris, France, May 1982.

[12] M. Pätzold, Mobile Radio Channels, John Wiley \& Sons, Chichester, England, 2nd edition edition, 2011.

[13] M. Pätzold and B. Talha, "On the statistical properties of sum-of-cisoidsbased mobile radio channel simulators," in Proceedings of the 10th International Symposium on Wireless Personal Multimedia Communications, WPMC 2007, pp. 394-400, Jaipur, India, 2007.

[14] I. S. Gradshteyn and I. M. Ryzhik, Table of Integrals, Series, and Products, Elsevier Academic Press, 7th edition edition, 2007.

[15] R. H. Clarke, "A statistical theory of mobile-radio reception," Bell Labs Technical Journal, vol. 47, no. 6, pp. 957-1000, 1968.

[16] M. Pätzold, C. A. Gutiérrez, and N. Youssef, "On the consistency of non-stationary multipath fading channels with respect to the average doppler shift and the doppler spread," in Proceedings of the 2017 IEEE Wireless Communications and Networking Conference, WCNC 2017, San Francisco, CA, USA, March 2017. 
[17] M. Pätzold, B. O. Hogstad, and N. Youssef, "Modeling, analysis, and simulation of MIMO mobile-to-mobile fading channels," IEEE Transactions on Wireless Communications, vol. 7, no. 2, pp. 510-520, 2008.

[18] M. Pätzold and C. A. Gutiérrez, "Enhancing the Resolution of the Spectrogram of Non-Stationary Mobile Radio Channels by Using Massive MIMO Techniques," in Proceedings of the 2017 IEEE 86th Vehicular Technology Conference (VTC-Fall), Toronto, Canada, September 2017. 


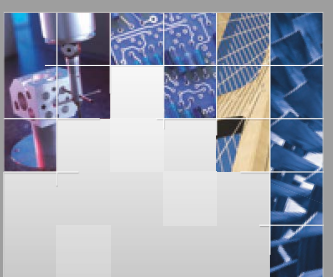

\section{Enfincering}
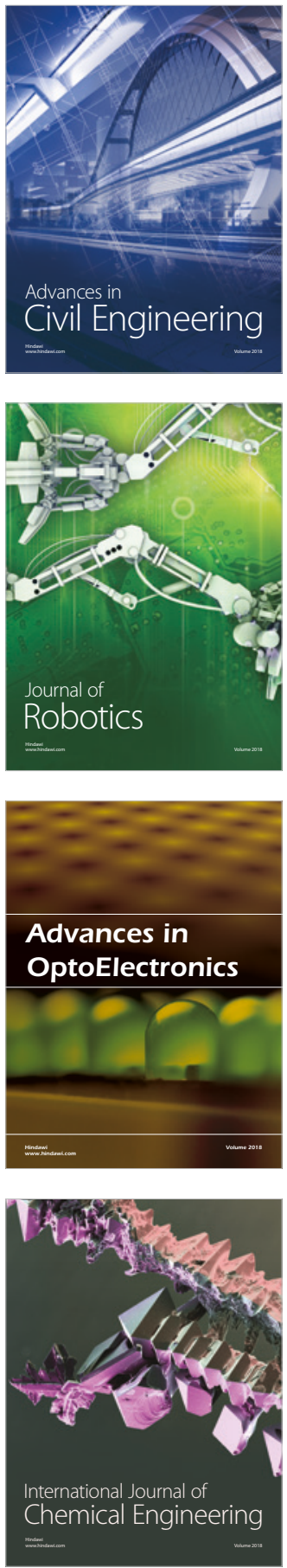

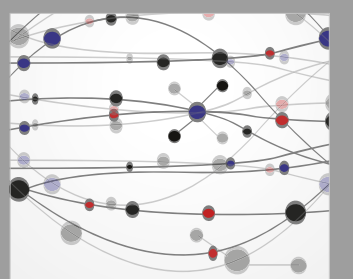

\section{Rotating \\ Machinery}

The Scientific World Journal

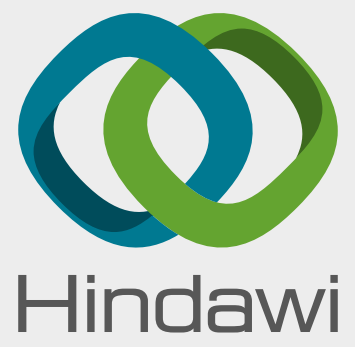

Submit your manuscripts at

www.hindawi.com
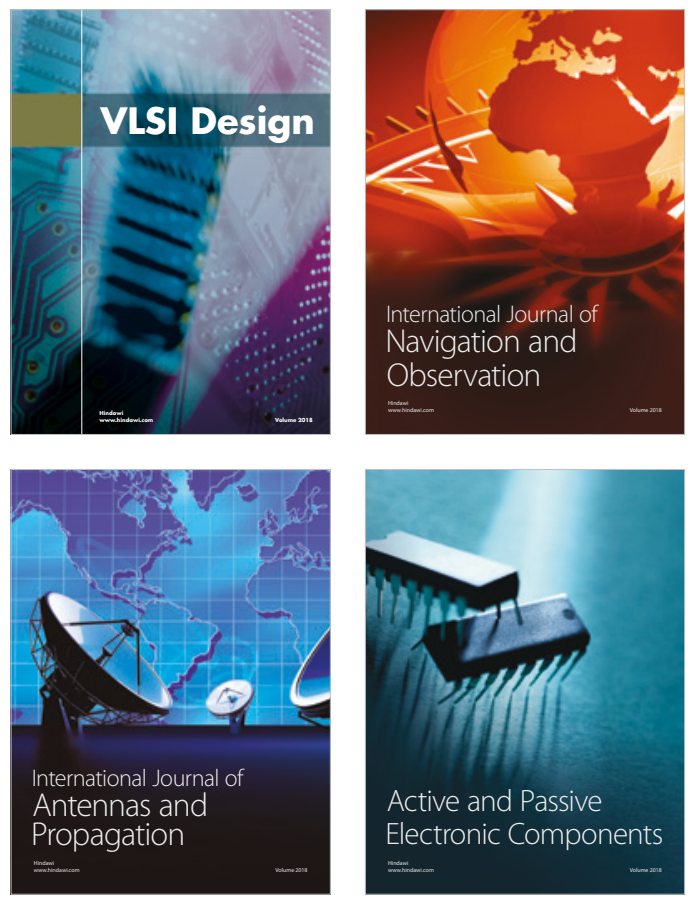
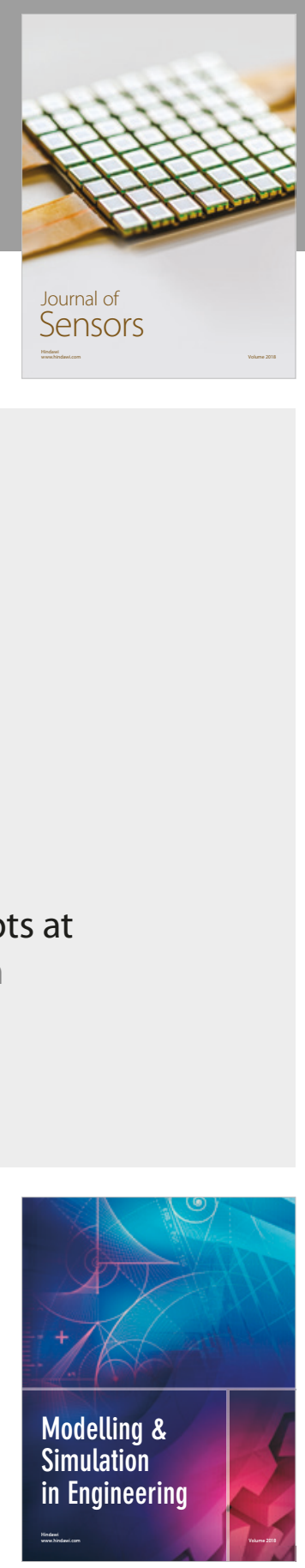

\section{Advances \\ Multimedia}
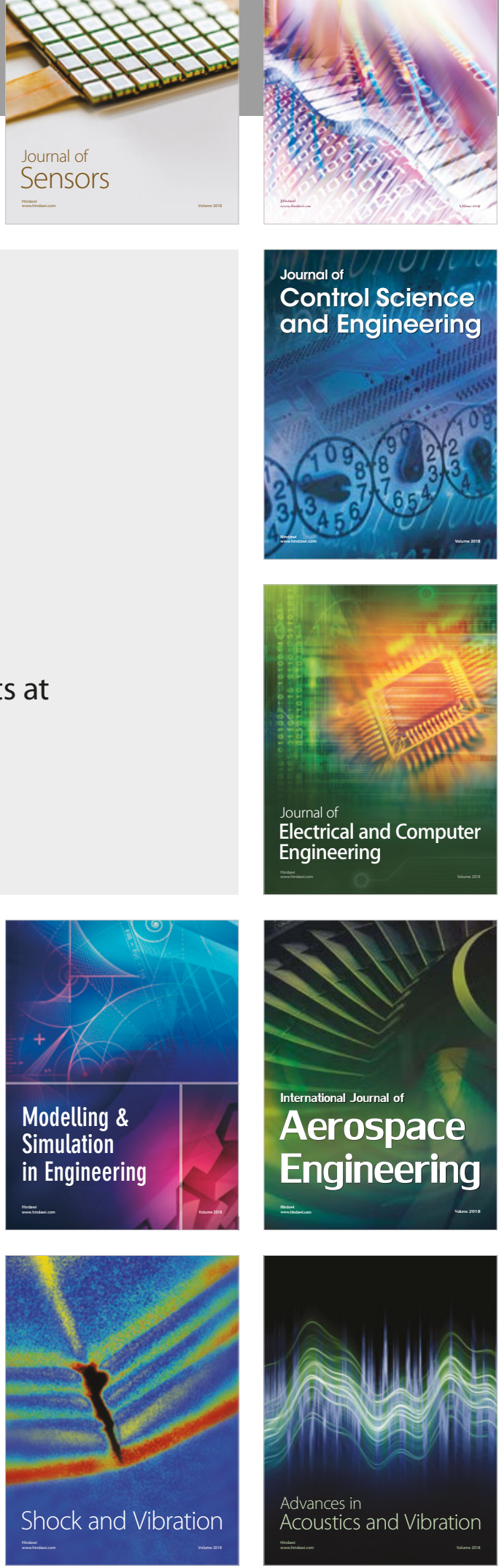